\title{
Bilderwechsel in der Sowjetunion: Freundbilder, Feindbilder, Standbilder, Ikonen
}

\author{
von Paul Roth
}

Jede Zeit, jede Gesellschaft hat ihre 'Bilderkollektionen': Freund- und Feindbilder, Standbilder und Ikonen (geistlicher und weltlicher Art). In pluralistischen Systemen stehen sie neben- und gegeneinander. Derjenige, der von einem Massenmedium oder einem Politiker als 'Betrüger', 'Trottel' oder 'Papiertiger' bezeichnet worden ist, kann sich wehren.

In totalitären, diktatorischen Regimen beanspruchen die Machthaber das 'Bildermonopol'. Sie bestimmen, wer verherrlicht, wer verdammt wird, wer als Vorbild gilt oder als Volksfeind. Dieses 'Bildermonopol' beschränkt sich nicht auf die Gegenwart, es bezieht die Vergangenheit mit ein. Die Veränderungen in der Gegenwart können dazu zwingen, auch die Vergangenheit zu korrigieren. Orwell hat in seinem Roman '1984' ein Regime beschrieben, das ständig mit der Korrektur der Vergangenheit befaßt ist und eine der Parteiparolen der KPdSU lautet: „Wer die Vergangenheit beherrscht, beherrscht die Zukunft; wer die Gegenwart beherrscht, beherrscht die Vergangenheit."

\section{Der Abbau von Lenin-Denkmälern}

In der Sowjetunion hat ein großes Abhängen und Aufhängen von Bildern begonnen; selbst die 'Ikonen' des Regimes sind nicht mehr sicher. Im April 1989 wagte ein Theaterregisseur aus Moskau, in einer TV-Live-Sendung folgenden Vorschlag zu machen: Entfernt Lenins einbalsamierte Leiche aus dem Mausoleum, beendet den heidnischen Brauch, gebt ihm ein richtiges Grab. Der Skandal, der sich daran anschloß, war so groß, daß der Verantwortliche für Hörfunk und Fernsehen, der etwa im Range eines Ministers steht, bald danach 'aus Gesundheitsgründen' abtrat. Es hat nicht viel geholfen. Nicht nur in den ehemaligen sozialistischen Bruderstaaten werden Lenin-Denkmäler abgebaut, auch in der Sowjetunion hat der Abbau begonnen. In Kaunas (Kowno) in Litauen wurde Anfang August 1990 auf Anordnung der städtischen Behörden das LeninDenkmal demontiert. Bald darauf ließen die örtlichen Behörden in Tscherwonograd und Ternopol in der Ukraine ebenfalls die Lenin-Denkmäler entfernen.

Das zentrale Parteiorgan 'Prawda' gab einem Artikel vom 05.08.1990 die Überschrift „Wissenschaft des Hasses?". In diesem Artikel ging es um den Aufschrei überzeugter Kommunisten, die ihre Welt zusammenbrechen sehen. Die 'Prawda' zitiert: „Die Perestroika hat eine Scheußlichkeit an die Oberfläche gebracht, die so giftig ist, daß es für sie nichts Heiliges im Leben mehr gibt ..." und: „Die schreckliche Stunde der Prüfung hat geschlagen. Im Lande herrscht gewal-

Dr. Paul Roth ist Professor für Politikwissenschaft und Publizistik an der Hochschule der Bundeswehr in München und Lehrbeauftragter an der Universität München, sowie anerkannter Experte zur Medienpolitik der Sowjetunion vor und nach der Perestroika. 
tige Verwirrung, breitet sich die Schmähung auf unsere Heiligtümer, auf Ehre und Würde aus ..... Der Verfasser des letzten Zitats, ein Oberst im Ruhestand, weiß auch, wer daran die Schuld trägt: der Zionismus.

Lenin ist sozusagen die Spitze der Bilderpyramide, die sich über Standbildern, Freund- und Feindbildern auftürmt. Die Glasnostpolitik Gorbatschows hat das Bildermonopol beiseite geschoben, die Pyramide ist ins Wackeln geraten. Daß damit auch Lenin ins Wackeln geriet, hat Gorbatschow weder beabsichtigt noch ahnen können. Wenn man sich die Geschichte der Sowjetunion anschaut, stellt man allerdings fest, daß es mehrfach 'Bilderwechsel' gegeben hat; nur waren die bisherigen von oben angeordnet. Der jetzige Bilderwechsel entzieht sich teilweise dem Bildermonopol der Machthaber.

\section{Das Freund - Feindbild der Bolschewiki}

Wie waren die Freund - Feindbilder ganz zu Beginn der Sowjetmacht? Die revolutionären Bolschewiki hatten diesbezüglich ganz klare Vorstellungen, auch wenn nur ihre Führung Marx und Engels gelesen hatte. Lenin hat sich die Lehre von Marx und Engels für seine politischen Ziele zurechtgelegt, - in der Sowjetunion sagte man: „Er hat sie fortentwickelt.“ Die Geschichte, so meinte er, sei eine Kette von Klassenkämpfen, in denen die Ausgebeuteten sich immer wieder gegen die Ausbeuter erheben. Jetzt, im 20. Jahrhundert, kämpfe das Industrieproletariat gegen die Ausbeuter, angeführt durch die Partei. Der 'Klassenfeind' war nicht nur der Feind der Arbeiter, des Volkes, sondern auch des Fortschritts und des Friedens in der Welt. Er war schlechthin 'das Böse'. Auf kommunistischen Plakaten der Revolutionsjahre war 'das Böse' in einer Dreifaltigkeit dargestellt: Zar, Kapital und Kirche. Bisweilen wählte man auch das Symbol der Schlange, die vom 'Ritter Georg' - in diesem Fall war das das Proletariat getötet wird.

Anfangs war das Denken der Revolutionäre noch internationalistisch, $\mathrm{d}$. h. der Klassenfeind existierte zwar im eigenen Land, gehörte jedoch zur Verschwörung der Klassenfeinde in der ganzen Welt. Ihm trat als Retter und Erlöser das revolutionäre, internationale Proletariat gegenüber. Der Klassenkampf mußte so lange geführt werden, bis der Klassenfeind auf der ganzen Welt besiegt war. Die Freunde und Verbündeten in diesem Kampf waren die Proletarier aller Länder und die unterjochten Kolonialvölker. Der Satz aus dem 'Kommunistischen Manifest' von Karl Marx „Proletarier aller Länder vereinigt euch“ steht bis heute im Kopf des Parteiorgans 'Prawda'.

In einem solchen Kampf durfte der Klassenfeind nach der Überzeugung Lenins keinerlei Möglichkeit haben zu überleben. Das Leninsche Dekret über die Presse war ein Unterdrückungsdekret. ${ }^{1}$ Lenin rechtfertigte dies mit den Worten: „Es ist unmöglich, die Pressefreiheit von den anderen Fragen des Klassenkampfes zu trennen." Damit war im Bereich der Medien jene 'tabula rasa' geschaffen, auf die die Bolschewiki ihre Aufrufe zur Vernichtung des Klassenkampfes ungestört schreiben konnten. 


\section{Die heiligste Stätte in Moskau}

Kampf gegen den Klassenfeind bedeutete jedoch nicht nur die Unterdrückung der bürgerlichen Presse, den Sieg über die 'weißen' Armeen, die Ermordung der Zarenfamilie, die Enteignung des Kapitals, sondern auch den Kampf gegen die Religion, gegen die Kirchen, gegen die christlichen Symbole in der Öffentlichkeit.

Wer heute auf dem Roten Platz in Moskau steht und die schwarzen Limousinen der politischen Führung aus dem Erlösertor herauskommen sieht, sollte sich daran erinnern, daß dieses Kremltor seinen Namen von einer Ikone hat, die 1647 daran angebracht worden war. Kein Mann durchschritt einst dieses Tor mit bedecktem Haupt. Die heiligste Stätte auf dem Roten Platz war die Kapelle der Iberischen Muttergottes (neben dem Historischen Museum). Wenn der Zar nach Moskau kam, suchte er die Kapelle auf, bevor er den Kreml betrat. Der „Rußland-Baedecker" vom Jahre 1912 schreibt: „Die Kapelle ist meist dicht umdrängt, auch nachts; man hüte sich vor Taschendieben." ${ }^{2}$ Die Erlöserikone am Kremltor verschwand rasch, die Kapelle der Iberischen Muttergottes wurde 1923 geschlossen.

Nach dem Tode Lenins im Jahr 1924 wurde zwischen den Gräbern der Revolutionäre, die für die Sowjetmacht gefallen waren, ein hölzernes Mausoleum auf dem Roten Platz errichtet. Als im Winter 1917 die im Kampf um die Macht in Moskau gefallenen Bolschewiki in Massengräbern vor der Kremlmauer beigesetzt wurden, war der amerikanische Journalist John Reed Augenzeuge. Er schrieb darüber: „Plötzlich wurde mir klar, daß das fromme russische Volk keine Priester mehr brauchte, um sich das Himmelreich zu erflehen. Auf Erden bauten sie an einem Reich, schöner, als es der Himmel sein konnte, und für ein solches Reich lohnte es sich, zu sterben." ${ }^{3}$

Lenin hatte sich in seinem Leben stets gegen die Verherrlichung seiner Person gewandt und ließ nicht einmal eine Briefmarke mit seinem Kopf zu. Die Erben der Macht entschieden sich aber für die Einbalsamierung des Leichnams, eine wahrlich seltsame Entscheidung von Materialisten, gegen die Lenins Witwe vergeblich protestierte. 1929 wurde beschlossen, das derzeitige Mausoleum zu errichten und 1934 erklärte eine Regierungsmission, daß das Problem der langanhaltenden Bewahrung des Körpers von Lenin als „blendend gelungen“ bezeichnet werden könne. ${ }^{4}$ Im selben Jahr begann Stalins Säuberung, die Jagd auf die 'Klassenfeinde' im Sowjetland.

Das Mausoleum Lenins ist sozusagen das 'Allerheiligste' der Sowjetunion. Vor ihm vollziehen sich die Demonstrationen, neben ihm wurden und werden jene beigesetzt, die sich um den Sowjetstaat verdient gemacht haben. Wenn keine Besucherströme an Lenins Leichman vorüberziehen und das Mausoleum geschlossen ist, steht doch die Tür zum Roten Platz eine Handbreit offen. Dies dient nicht zur Lüftung, sondern ist symbolisch zu verstehen.

\section{Lenins Monumentalpropaganda}

Im vorrevolutionären Moskau gab es über 500 Kirchen und Kapellen, aber nur 10 Denkmäler. Während nach der Revolution die Kirchen geschlossen, 
aber noch nicht zerstört wurden, begann eine eifrige Vermehrung der Denkmäler. Am 4. April 1918 lud Lenin den Volkskommissar für Bildung, A. W. Lunatscharski, zu sich ein und erklärte ihm: „Schon lange schwebte mir diese Idee vor, die ich Ihnen gleich erklären werde. .. Sie erinnern sich, daß Campanella in seinem 'Sonnenstaat' davon spricht, daß auf den Mauern seiner phantastischen sozialistischen Stadt Fresken aufgemalt sind, die der Jugend als anschaulicher Unterricht für Naturwissenschaft, Geschichte dienen können, die das BürgerGefühl wecken - kurz, teilnehmen an der Bildung, der Erziehung neuer Geschlechter. Mir scheint, daß es durchaus nicht naiv ist, und mit gewissen Veränderungen könnte das jetzt von uns angeeignet und verwirklicht werden."

'Monumental-Propaganda' oder 'Propaganda durch Skulpturen' ist dieser Vorschlag genannt worden. W. Majakowski schrieb: „Die Straßen sind unsere Pinsel, die Plätze unsere Paletten." Eine erste Liste jener Männer und Frauen, die durch Denkmäler geehrt werden sollten, umfaßte 50 Personen alleine für Moskau: Revolutionäre, Philosophen, Künstler aus der ganzen Welt. Nur ein Teil davon wurde verwirklicht. Viele verschwanden, da sie aus ungeeignetem Material geschaffen worden waren. Andere wiederum, die im kubofuturistischem Stil errichtet worden waren, fielen später dem 'sozialistischen Realismus' zum Opfer.

Als Lenin starb, waren die Freund- und Feindbilder klar, ebenso die Vorbilder. In Karikaturen und auf Plakaten konnte man sie sehen. Die Embleme der Zarenzeit waren abgeschlagen und durch den Sowjetstern ersetzt worden. Auf den Wänden wurde verkündet, was das Volk wissen sollte, so z. B. „Religion ist Opium für das Volk" oder "Wer nicht arbeitet, der ißt auch nicht."6 Bemerkenswert ist, daß unter den Vorbildern kein Lebender war und daß ihre Auswahl nicht unter nationalistischem Gesichtspunkt getroffen worden war. Allerdings waren die großen Symbolfiguren der damaligen sowjetischen Briefmarken eindeutig Sowjetbürger: Arbeiter, Bauern, Rotarmisten (und erst 1929 wird dem Arbeiter eine Arbeiterin sowie dem Bauern eine Bäuerin zur Seite gestellt.)

\section{Stalins Bilderkollektion}

Unter Stalin begann ein Bilderwechsel. Dies hing sowohl mit seiner Säuberung zusammen als auch mit dem Sowjetpatriotismus, der nun dem Marxismus-Leninismus beigemengt wurde. Als Stalin die 'Neue Ökonomische Politik' Lenins abwürgte und die Zwangskollektivierung durchführte, waren die Zeitung und Mauern überschwemmt mit den übelsten Hetzbildern von 'Spekulanten' und 'Kulaken'. Die logische Folge der Behauptung, daß in der Sowjetunion keine Klassengegensätze mehr bestünden, war die Gleichsetzung des 'Klassenfeindes' im Sowjetland mit dem 'Volksfeind'. Dieser 'Volksfeind' wiederum wurde beschuldigt, im Dienst auswärtiger, kapitalistischer Mächte zu stehen. Wolfgang Leonhard schildert seine Bestürzung, als er am 12.06.1937 in der 'Prawda' auf fünf von sechs Seiten Schlagzeilen findet, die jeweils mit der Aufforderung endeten: „Erschießt sie.“7 
Es war eine durch und durch verlogene 'Bildersprache'. Stalin scheute nicht davor zurück, alte Kampfgenossen Lenins heimlich und öffentlich umbringen zu lassen. Der Parteisekretär von Leningrad, Kirow, den er heimlich hatte ermorden lassen, wurde gleich in die Heldengalerie eingereiht, mit Denkmälern und Briefmarke geehrt. Viele Mitstreiter Lenins, die Stalin umbringen ließ, wurden zu Ungeheuern und bezahlten Söldlingen erklärt und mußten in Schaugerichtsprozessen ihre 'Verbrechen' gestehen. Stefan Heym hat dies in seinem Roman „Der König David Bericht" verfremdet dargestellt. Der einstige Kriegsheld Davids, Joab, muß sich folgende Sätze anhören: „Du selbst gehörtest einst zu den Mächtigen im Königreich, und zu denen, die entschieden, ob einer gewaltsam zu Tode gebracht wird oder ob er seine Tage in Frieden beschließen darf. Ich aber entscheide, wie einer weiterlebt nach seinem Tode, in den Augen künftiger Geschlechter, und ob er in tausend Jahren als ein kindischer Greis gilt, dem der Speichel in den Bart rinnt und die Pisse in die Hosen, oder als ein Soldat, der seinem Schicksal mit Würde und Mut ins Antlitz blickt. ${ }^{\text {"8 }}$

Die Karikaturisten hatten ihre festen Schablonen für diese 'Volksfeinde' und 'Schädlinge', diesen 'Abschaum der Menschheit', die 'Kriegstreiber' und ihre 'Spione' im Sowjetland. Hand in Hand mit dem Gottlosenverband schmähten sie alle Gläubigen. Die massenhafte Zerstörung von Kirchen sollte nicht nur die 'Brutstätten des Aberglaubens' beseitigen, sondern auch die Stadtbilder verändern, die von Kirchtürmen überragt waren. Nach der Sprengung der ErlöserKathedrale in Moskau sollte an derselben Stelle der Palast der Sowjets errichtet werden, gekrönt von einer riesigen Leninstatue. Der Plan wurde aber nicht verwirklicht, denn angeblich war der Untergrund nicht fest genug für das riesige Monument.

1933 hatte der Akademiker Strumilin geschrieben: „Wenn man über die Architektur Moskaus spricht, so soll man sich daran erinnern, daß es die Hauptstadt der UdSSR und der Stab der Komintern ist. ${ }^{49}$ Im Jahr darauf entschied die Moskauer Parteiorganisation: „Die Gestaltung der Stadt muß die Erhabenheit und Schönheit der sozialistischen Epoche widerspiegeln." Zu dieser Zeit wurden solche Worte und Sätze geschrieben, weil Stalin seine 'Wunsch-Befehle' hatte wissen lassen.

Stalin hat die Geschichte der Sowjetunion umschreiben lassen, so daß auf einmal Peter der Große und Iwan der Schreckliche große Gestalten der Vergangenheit waren. Im 'Großen Vaterländischen Krieg' trat eine Nationalhymne an die Stelle der Internationale, die bis dahin Staatshymne gewesen war. Das Freund-Feindbild-Klischee der Vergangenheit wurde umgemodelt. Nicht mehr der Internationalismus, sondern der Sowjetpatriotismus setzte die Akzente. Ob jemand als Feind dargestellt wurde, hing nur noch davon ab, ob er sich gegenüber der Sowjetunion feindlich oder ablehnend verhielt. Nach dem Hitler-StalinPakt des Jahres 1939 waren Feinddarstellungen des deutschen Faschismus in der Sowjetunion verboten. Man regte sich nicht mehr darüber auf, daß die Kommunisten in Hitler-KZs gequält und ermordet wurden. Kommunisten, die sich in die Sowjetunion geflüchtet hatten, wurden teilweise an Hitler ausgeliefert. 
Nach dem Überfall Hitlers auf die Sowjetunion kam die patriotische Wende auch der Russisch-Orthodoxen Kirche zugute. Sofort nach dem Angriff wurde die Gottlosenpropaganda eingestellt. Dimitri Donskoi und Alexander Newski, die einst mit Christusbannern über ihren Heerscharen in den Kampf gegen die Feinde Rußlands gezogen waren, wurden als große Vorbilder herausgestellt. Auf dem Moskauer U-Bahnhof 'Komsomolskaja' erblickt man über sich im glänzendem Mosaik das Christusbanner.

Nach dem Krieg und nach dem Bruch der Anti-Hitler-Koalition legte die politische Führung die neuen Feinde der Sowjetunion, des Weltfriedens usw. fest: die USA, Großbritannien, bald auch die Bundesrepublik Deutschland und den 'Zionismus'. Die höchste Form der Verunglimpfung in Karikaturen und auf Plakaten bestand darin, daß man diese 'Feinde' in irgendeiner Form mit Hitler oder Goebbels in Verbindung brachte.

Alles sollte unter Stalin größer und heroischer sein, als es je zuvor gewesen war. Die Revolutions-Denkmäler traten gegenüber den Sieges-Denkmälern zurück, denn schließlich hatte Stalin in der Oktoberrevolution nur eine untergeordnete Rolle gespielt. Die Symbolfiguren der Leninzeit - Arbeiter, Bauern, Rotarmisten - wurden auf Briefmarken abgebildet. Für die Pariser Weltausstellung am Vorabend des Zweiten Weltkrieges kam aus der Sowjetunion eine Monumentalplastik von Wera Muchina: Arbeiter und Bäuerin reckten Hammer und Sichel in den Himmel. Danach wurde die gigantische Plastik in der Moskauer Allunionsausstellung aufgestellt. Heute weiß man nicht so recht, was man mit ihr anfangen soll, sie beginnt zusammenzubrechen.

Moskau wurde nach dem Krieg mit einigen Wolkenkratzern verunstaltet, die man in der Chruschtschow-Zeit spöttisch als Zuckerbäckerstil-Erzeugnisse bezeichnete. Bis heute überragen sie die Hauptstadt als Hotel, Außenministerium und Universität. Die Ideologie fordert - trotz aller 'Fortentwicklung' - eine Kontinuität. $\mathrm{Zu}$ dieser Kontinuität gehörte der fleißig gepflegte Lenin-Kult. Überragt wurde er allerdings durch den Stalin-Kult. Besonders schön kann man das an Demonstrationsbannern und Büchern jener Zeit erkennen: Die Köpfe von Marx-Engels-Lenin und Stalin sind in einem ovalen Rahmen neben- und übereinander aufgereiht, obenauf selbstverständlich Stalin.

\section{Chruschtschows Entstalinisierung}

Der Tod Stalins im Jahr 1953 erschütterte die Sowjetunion und die Sowjetbürger nachhaltig. Jahrzehntelang war ihm gehuldigt worden wie einem Gott - und nun hatte der Gott sich als sterblich erwiesen. Einbalsamierung und Beisetzung an der Seite Lenins erschienen damals als die einzig würdige Wahrung des Andenkens. 1954 erschien zum letztenmal eine Briefmarke mit der Darstellung Stalins; das 'Tauwetter' hatte behutsam begonnen.

Wenden wir uns aber erst den 'Kontinuitäten' zu. Für die 'Feinde' und 'Schädlinge' in der Sowjetunion verwendete man nicht mehr den 'Volksfeind'Begriff aus der Stalinzeit. Wenn auf Mängel und Verfehlungen hingewiesen 
wurde, wenn Karikaturen und Plakate sich damit befaßten, so geschah das nach Anweisung von oben und unter Vermeidung jeder 'Verallgemeinerung', $\mathbf{d}$. h. Fehler und Mängel, Trunkenbolde, bestechliche Beamte, unfähige Direktoren usw. durften immer nur als Ausnahmen, als bedauerliche Einzelfälle dargestellt werden. Keinesfalls durfte der Eindruck entstehen, es handele sich um Exemplare des 'neuen kommunistischen Menschen'.

Für Feinde im Ausland wurde das Schema aus der Stalinzeit übernommen. Aufgespießt und angeprangert wurden jene, die sich der Sowjetunion gegenüber zurückhaltend, ablehnend oder feindlich verhielten. Nicht angegriffen wurden jene Staaten und Staatsmänner, die zur Sowjetunion freundschaftliche Beziehungen unterhielten, wobei es keine Rolle spielte, ob in diesen Staaten Kommunisten verfolgt wurden oder nicht. Außerhalb der Kritik befanden sich die 'sozialistischen Bruderländer'. Jeder Vergleich zwischen der DDR und der Bundesrepublik Deutschland fiel zugunsten der DDR aus.

Als Chruschtschow 1956 auf dem XX. Parteitag mit der Entstalinisierung begann, mußte die 'Heldengalerie' umsortiert werden. Stalinbilder und Stalindenkmäler verschwanden. Nach dem XXI. Parteitag 1961 wurde Stalins Leichnam aus dem Mausoleum entfernt. Man hielt ihn nicht mehr für würdig, neben Lenin zu ruhen. Immerhin erhielt der tote Diktator einen Ruheplatz neben dem Mausoleum - zuerst nur mit liegender Platte. Als die Entstalinisierung nach Chruschtschows Zwangspensionierung im Jahr 1964 eingestellt wurde, bekam Stalin auch eine Büste aufs Grab. Wo allerdings Stalins Oberhenker Berija nach der Hinrichtung verscharrt wurde, ist unbekannt.

Stalingrad wurde in Wolgograd umbenannt, der Stalinpreis in Leninpreis. Wer mit dem Stalinpreis ausgezeichnet worden war, mußte die Medaille zum Umtausch abliefern und erhielt eine neue Medaille mit Leninkopf. Opfer der Säuberungen Stalins wurden rehabilitiert. So wurde z. B. der 1937 erschossene Marschall Tuchatschewski 1963 durch eine Briefmarke geehrt. 1963 geriet jedoch die Entstalinisierung bereits ins Stocken.

Stalin hatte bei Ausbruch des Krieges die Gottlosenpropaganda einstellen lassen. Seit 1947 wurde sie von der 'Gesellschaft zur Verbreitung wissenschaftlicher und politischer Kenntnisse' sehr gedämpft wieder aufgenommen. Chruschtschow - und dies gehört wieder zum Thema 'Bilderwechsel' - leitet 1958/59 eine neue rüde Verfolgung der Religionsgemeinschaften ein. Den Massenmedien wurde befohlen, den Kampf gegen die Religionsgemeinschaften wieder aufzunehmen. In Moskau erschien ein atheistisches Journal und Karikaturisten und Plakatmaler beteiligten sich - in Anlehnung an die Vergangenheit an dieser Aktion.

\section{Die Zeit der 'Stagnation'}

Breschnew übernahm von Chruschtschow 1964 eine Sowjetunion, die übersät war mit Lenin-Denkmälern und mit Gedenkstätten für den 'Großen Vaterländischen Krieg'. ${ }^{10}$ Die Gedenkstätten für die Oktoberrevolution traten dem- 
gegenüber in den Hintergrund. Im Rückblick auf die Breschnew-Zeit spricht man heute von der 'Zeit der Stagnation'. Das läßt sich durchaus auch für die Darstellung von Freund und Feind übertragen.

Die 'Säufer', 'Bummelanten', 'Schädlinge' usw. im eigenen Land wurden weiter als Ausnahmen, als Einzelfälle angeprangert. Die Feinde im Ausland die 'Imperialisten', 'Kriegstreiber' und 'Friedensfeinde' - wurden weiter mit der Elle gemessen, wie ihr Verhältnis zur Sowjetunion war. Nach dem Bruch zwischen Moskau und Peking wurden nun auch die kommunistischen Führer in China von Karikaturisten verhöhnt, und nach dem Einmarsch der Sowjetunion in Afganistan (1979) wurden die amerikanischen Imperialisten als die eigentlichen Feinde der 'Volksmacht' in Afghanistan angeprangert. Sie unterstützten so behaupteten es die sowjetischen Medien und zeigten es in Karikaturen und Plakaten - Halsabschneider, Mörder und Banditen in Afghanistan.

'Unruhestifter' in der Sowjetunion - gemeint sind die Dissidenten - wurden als vom Ausland gekaufte Subjekte diffamiert. Solchenizyn wurde ausgewiesen, Sacharow nach Gorki verbannt, andere wurde als Geisteskranke bezeichnet. Breschnew, der offensichtlich von einer greisenhaften Eitelkeit besessen war, ließ sich ständig neue Orden und Titel verleihen, darunter sogar den Leninpreis für Literatur und den Marschalltitel für Heldentaten im 'Großen Vaterländischen Krieg'. Immerhin dämpfte er aber die Verfolgung der Religionsgemeinschaften.

In den letzten Lebens- und Herrschaftsjahren des Greises nahm man seinen Personenkult auch in der Bevölkerung nicht mehr ernst. Seine kurzlebigen Nachfolger - Andropow und Tschernenko - hätten wohl an der Bildergalerie noch etwas verändert (so war eine riesige Kriegsgedenkstätte in Moskau geplant), aber ihr frühzeitiger Tod verhinderte solche Pläne.

\section{Gorbatschows Übergangsphase}

Die Perestroika und Glasnost-Politik Gorbatschows, vor allem aber das 'Neue Denken' in der sowjetischen Außenpolitik, haben zu einem fortgesetzten Abhängen und Aufhängen von 'Bildern' geführt. Allerdings war dieser Prozeß, wie er sich vor allem seit 1987 entwickelt hat, von Gorbatschow im Jahre 1985 durchaus nicht so beabsichtigt. Anfangs, in den Jahren 1985 und 1986, ging es Gorbatschow um die Perestroika im Wirtschaftsbereich, nicht um die Aufarbeitung der Vergangenheit, schon gar nicht um die Freisetzung einander bekämpfender 'Feindgruppen' in der Sowjetunion selber.

Die vorsichtige Zurücknahme der Zensur in den Jahren 1985 und 1986 gab zunächst das Schußfeld frei für alle jene, die der Veränderung der Wirtschaft im Wege standen. Überprüft man die Negativ-Figuren jener Jahre, so sind es bereits altvertraute Gestalten: Säufer, Bummelanten,unfähige Direktoren, diebische Funktionäre usw. Die Karikaturen und die Anprangerungen unterschieden sich kaum von jenen in den zurückliegenden Jahren. Nur der aufmerksame Beobachter konnte den Schluß ziehen, daß die Fülle einen Umschlag der 'Quanti- 
tät' in 'Qualität' andeutete. Mit anderen Worten: Es handelte sich offensichtlich nicht nur um Einzelfälle, sondern um gesellschaftliche Mißstände.

Eine Auseinandersetzung mit der Vergangenheit wies Gorbatschow damals mit den Worten zurück, daß es dafür noch zu früh sei. Als 1986 in der 'Prawda' ein Leserbrief veröffentlicht wurde, der die Privilegien der Nomenklatura angriff, bekam die 'Prawda' einen Rüffel, und als im gleichen Jahr die Tschernobyl-Katastrophe zuerst verheimlicht, dann heruntergespielt wurde, schien 'Glasnost' wieder beiseitegerückt zu sein. Wieder schien es, als hätten nur einzelne versagt.

1985 und 1986 war auch das Feindbild von den Imperialisten, den Kriegstreibern im Ausland noch unverändert. Der Krieg in Afghanistan wurde weiter als mutiges Eintreten der Sowjetunion, als 'Erfüllung einer internationalistischen Pflicht' dargestellt. Kritik an den sozialistischen Bruderstaaten war weder erwünscht noch zugelassen.

\section{Bilderwechsel in den Medien}

Seit 1987 begann sich das Medienmonopol, das Bildermonopol der politischen Führung jedoch zu zersetzen. Zuerst geschah das vorsichtig, zögerlich, dann immer rascher. Die offizielle 'Glasnost', die als obrigkeitlicher Akt begonnen hatte, sah sich mehr und mehr einer eigenständigen 'Glasnost' gegenüber.

Die Massenmedien, vor allem die Printmedien, waren bis dahin immer als Instrumente der Führung gehandhabt worden, die gehorsam und schnell alle Befehle der Obrigkeit umsetzten. Seit 1987 begannen sich vor allem aber die Printmedien in verschiedene Richtungen auseinanderzuentwickeln. Die einen vertraten eine konservative Richtung, wälzten die Schuld auf Breschnew und Stalin $\mathrm{ab}$, wollten jedoch nur eine kosmetische Veränderung. Keinesfalls sollte z. B. die Führungsrolle der Partei angetastet werden. Musterbeispiele für diese Richtung waren die 'Prawda', die 'Sowjetskaja Rossija' und die 'Krasnaja swesda'.

Andere wiederum verlangten eine Beschleunigung des Perestroika-Prozesses in Richtung auf einen liberalen Rechtsstaat. Sie schreckten auch nicht davor zurück, die Geschichte der Sowjetunion neu aufzuarbeiten. $\mathrm{Zu}$ dieser Richtung gehörten u. a. die 'Iswestija', die 'Komsomolskaja prawda' (mit ihrer Jugendbeilage 'Sobesednik'), 'Argumenty i fakty' und die 'Moskowskie nowosti'. Ähnlich war das Auseinanderdriften bei den Zeitschriften, vor allem bei den 'dicken', literarischen Zeitschriften. Während z. B. 'Molodaja gwardija' und 'Sowremennik' bremsten, wagten sich 'Ogonjok' und 'Nowy mir' weit vor. Die Auseinandersetzung zwischen den Flügeln war heftig und ist bis heute heftig geblieben. Bei den Zeitschriften spricht man von einem 'Bürgerkrieg der Literaten'. Natürlich sind die Leit- und Vorbilder dabei völlig verschieden.

Gorbatschow hat verzweifelt versucht, diesen Streit, diese Aufspaltung zu mildern. So hat er z. B. im Herbst 1989 den Chefredakteur der 'Prawda' aus- 
gewechselt und meinte, nun könne man auch den Chefredakteur von 'Argumenty i fakty' austauschen. Der Chefredakteur von 'Argumenty i fakty' wehrte sich jedoch erfolgreich und blieb im Amt.

Sichtbarer und greifbarer war der Bilderwechsel im Theater, im Film und im Fernsehen. In Theaterstücken traten auf einmal Personen auf, deren Namen man vorher nur flüsternd genannt hatte (z. B. Trotzki). T. Abuladses Film 'Reue' nannte zwar an keiner Stelle den politischen Führer Stalin, aber jeder verstand, daß dieser Mörder und Henker auf der Leinwand Stalin sein sollte. Im Fernsehen wurden mehr und mehr Live-Sendungen gebracht, die nicht zensiert werden konnten. Mutige Redakteure zeigten den Zuschauern auf dem Bildschirm jene Mißstände, die zwar alle gekannt hatten, die jedoch nie gezeigt werden durften.

Als der Film 'Die kleine Vera' herauskam, war ein weiteres Tabu gebrochen. Er zeigte einen Geschlechtsakt. Von nun an bemühte sich fast jeder Filmund Fernsehregisseur in der Sowjetunion, in seinem Film oder Theaterstück wenigstens einmal einen Nackedei zu zeigen. Auf einmal gab man zu, daß es in der Sowjetunion eine weitverbreitete Prostitution gab. Dann fiel auch der Schutz der Nomenklatura: Die Sowjetbürger konnten die Verhaftung eines Ministers im Foto betrachten.

Die Zensur ist weiter zurückgenommen worden. Die Darstellung Stalins als Mörder wurde geradezu selbstverständlich, auch wenn man davor zurückschreckte, entsprechende Plakate in der Öffentlichkeit auszuhängen. In Plakatausstellungen gab es sie aber. Im Laufe der Zeit wagten sich einzelne Redakteure sogar bis Lenin vor. War er wirklich das Genie, der unfehlbare Revolutionär mit reinen Händen? Hatte er nicht mit der Anlage der KZs in der Sowjetunion begonnen? War er nicht mitschuldig an der Ermorderung der Zarenfamilie usw.?

Während dies alles sozusagen unter offizieller Duldung bzw. unter dem Schutzschild von Glasnost vor sich ging, griff der Samisdat immer ungenierter nach den Standbildern der Vergangenheit. Unter Samisdat versteht man jene Einzeltexte oder Periodika, die in Eigenverantwortung hergestellt und unter Umgehung der Zensur verteilt wurden. Der Samisdat stellt keinesfalls eine geschlossene Opposition dar. Es gibt Samisdat-Erzeugnisse, die rein religiös sind oder russisch-chauvinistisch. Aber ein Teil des Samisdat geht nicht nur völlig schonungslos mit Lenin um und bekämpft die Kommunistische Partei, er greift auch Gorbatschow und seine Politik frontal an. Er hat die 'alten Bilder' sozusagen auf den 'Misthaufen der Geschichte' geworfen.

\section{Feindbilder in der Sowjetunion}

Über viele Jahrzehnte hinweg war die Sowjetunion als ein gewaltiges Monument der Einigkeit dargestellt worden, angeführt von der Kommunistischen Partei. Die Klassengegensätze waren angeblich verschwunden, die Nationalitäten lebten angeblich in Frieden zusammen, die Sowjetmenschen hatten angeblich den Marxismus-Leninismus als einzig richtige Weltanschauung akzeptiert. 
Dieses Monument zerbröckelt jedoch seit 1988 unaufhaltsam. Informelle Gruppen bildeten sich, ein Teil davon betätigte sich politisch, Vorformen politischer Parteien entstanden, aus denen dann politische Gruppierungen und Parteien hervorgingen etc. Im Baltikum, im Kaukasus, in der Ukraine usw. entstanden Volksfronten, die Unabhängigkeit von Moskau forderten. Die Symbole der Kommunistischen Partei und der Sowjetunion wurden nicht mehr akzeptiert, man wählte sich eigene. Während es früher zum alljährlichen Ritual gehörte, daß das Sowjetvolk an seiner Führung geschlossen vorbeimarschierte und rief „Es lebe die KPdSU“, mehrten sich ganz andere Demonstrationszüge. Auf einmal gab es Transparente, die die Ablösung der Kommunistischen Partei forderten, Bilder des ermordeten Zaren oder Nationalfahnen usw. Doch wandte sich die Ablehnung nicht nur gegen die Herrschenden.

Feindbilder innerhalb der sowjetischen Bevölkerung tauchten auf. Russische Chauvinisten beschuldigten Juden, sie seien am Niedergang Rußlands schuld. Im Kaukasus bekämpften sich nicht nur christliche und muslimische Volksgruppen, sondern auch muslimische Volksgruppen untereinander. Die Zentralmacht griff mit Soldaten ein und zog sich teilweise den Haß der Nationalitäten zu.

Verzweifelt versuchte Gorbatschow, die Entstehung eines Mehrparteiensystems zu verhindern. Ende 1989 muß er aber eingesehen haben, daß das Herrschaftsmonopol der Kommunistischen Partei nicht mehr aufrechtzuerhalten war. Im Frühjahr 1990 verzichtete die Kommunistische Partei dann auf ihr Führungsmonopol und änderte die sowjetische Verfassung.

Inzwischen zeigte sich jedoch, daß es nicht nur verfeindete politische Gruppierungen in der Sowjetunion gab, sondern auch innerhalb der Kommunistischen Partei. Der XXVIII. Parteitag im Jahre 1990 wurde zwar nicht mit einer offiziellen Spaltungserklärung abgeschlossen, aber in Wirklichkeit hatte sich die KPdSU in mindestens drei Gruppen aufgespalten. Man nennt sie verhüllend 'Plattformen', obwohl es weitgehend unvereinbare Plattformen sind. Boris Jelzin trat mit anderen angesehenen Persönlichkeiten aus der Partei aus.

Das Monument ist im Zerfall. Die alten Bilder stimmen nicht mehr. Während man gelegentlich noch Plakate sieht „Lenin lebt, Lenin lebt, Lenin wird ewig leben", werden in einzelnen Städten die Lenin-Denkmäler abgebaut. Während z. B. die satirische Zeitschrift 'Krokodil' Stalin als Mörder darstellt, ragt nur wenige Meter vom Lenin-Mausoleum Stalins Grabstelle empor, als wäre er immer noch einer der Großen der sowjetischen Geschichte. Wenn man vom Roten Platz über das Mausoleum und die Kremlmauer blickt, sieht man dort eine unermüdlich flatternde rote Fahne, das Symbol der Sowjetmacht. Kaum jemand weiß, daß diese Fahne nur deswegen unermüdlich flattert, weil ein Gebläse für den Wind sorgt.

\section{Die Rücknahme der antireligiösen Propaganda}

Unerwartet und sensationell ist der Schwenk in der sowjetischen Religionspolitik unter Gorbatschow. Er wurde 1987/88 eingeläutet, als die Millen- 
niumsfeier der Taufe der Kiewer Rus bevorstand. Gorbatschow empfing den Patriarchen Pimen und das Moskauer Patriarchat konnte in Moskau mit großem Aufwand das Jubiläum feiern. Die bis dahin übliche atheistische und antireligiöse Propaganda verschwand aus den All-Unionsmedien und sogar aus dem atheistischen Journal 'Wissenschaft und Religion'. In Broschüren sind allerdings noch Reste zu finden.

Gorbatschow braucht für seine Perestroika die Mithilfe der Religionsgemeinschaften. Immerhin gibt es in der Sowjetunion rund 70 Millionen Gläubige verschiedener Konfessionen. Seit 1988 bringen sowjetische Medien immer häufiger Bilder von Kirchen, Priestern, religiösen Zeremonien usw. Es ist geplant, die Erlöser-Kathedrale in Moskau und die Kasan-Kathedrale, die einst am Roten Platz stand, wieder zu errichten. Woher man die Gelder nehmen will, ist allerdings unbekannt.

Die orthodoxen Kommunisten und die atheistischen Propagandisten sind in großer Verwirrung. Die Kommunistische Partei hält am MaterialismusAtheismus fest, aber sie stellt die Religionsgemeinschaften nicht mehr als Feind des sozialistischen Aufbaus dar. Es gilt die Parole: Gläubige und Nichtgläubige sitzen in einem Boot, dem Boot der Perestroika, und sollen die gleichen Rechte haben.

Während dieses Feindbild aus der Öffentlichkeit zurückgezogen worden ist, leben andere Feindbilder weiter, die innerhalb oder zwischen Religionsgemeinschaften bereits bestanden haben. Über die blutigen Kämpfe zwischen christlichen und muslimischen Volksgruppen bzw. innerhalb muslimischer Volksgruppen, ist bereits berichtet worden. Es gibt aber auch Feindbilder innerhalb der Russisch-Orthodoxen Kirche. Gruppen orthodoxer Gläubiger beschuldigen das Moskauer Patriarchat, daß es sich nach wie vor regimehörig verhalte. Das Moskauer Patriarchat wiederum wirft diesen Gruppen eine extremistischunchristliche Haltung vor.

Ungelöst ist der Konflikt zwischen der unierten katholischen Kirche in der Ukraine und dem Moskauer Patriarchat. 1946 hatte das Moskauer Patriarchat in Zusammenarbeit mit Stalin die Unierte Kirche in der Ukraine organisatorisch zerschlagen. Das Moskauer Patriarchat hat sich die Kirche der Unierten genommen. Seit 1990 dürfen in der Ukraine wieder unierte Gemeinden gegründet werden und Hunderte von Gemeinden des Moskauer Patriarchates haben sich den Unierten angeschlossen. Das Patriarchat weigert sich aber, die Aktionen des Jahres 1946 als rechtswidrig anzuerkennen und Wiedergutmachung zu leisten.

\section{Beseitigung außenpolitischer Feindbilder}

Die Beseitigung der außenpolitischen Feindbilder ist ebenfalls von oben in Gang gebracht worden. Es begann 1987 damit, daß offiziell zugegeben wurde, die Doktrin der 'friedlichen Koexistenz' (als spezifische Form des Klassenkampfes) sei überholt. Nicht mehr der Klassenkampf stehe an oberster Stelle, sondern die Bewahrung der allgemein-menschlichen Werte und des Friedens 
als Voraussetzung hierfür. Nach und nach gab man Fehler der sowjetischen Außenpolitik zu. Schließlich wurde sogar zugegeben, daß der Einmarsch in Afghanistan ein Fehler gewesen sei.

Gorbatschow brauchte für seine Perestroika die Zusammenarbeit mit den 'kapitalistischen' Staaten und wollte bzw. mußte die eigenen Rüstungskosten senken. Vertrauen konnte er im Ausland nur gewinnen, wenn das Feindbild des 'Imperialismus', der 'Kriegstreiber' usw. abgebaut wurde. Die Forderungen nach dem Abbau des gegenseitigen Feindbildes wurden wiederholt, die entsprechenden Karikaturen und Plakate verschwanden. Die sowjetischen Zentralmedien begannen mit einer Berichterstattung über die 'kapitalistischen' Staaten, die sich um Objektivität bemühte. Nicht nur der hohe Lebensstandard wurde zugegeben, auch die soziale und medizinische Versorgung der Bevölkerung wurde gelobt. 'Tele-Brücken' gewährten Sowjetbürgern einen kleinen Einblick in die fremde Welt; die Reisemöglichkeiten für Sowjetbürger wurden ausgedehnt und die Störung der Auslandssender eingestellt.

In der Diskussion über den Marxismus-Leninismus wurde zugegeben, daß die 'kapitalistischen' Staaten viele Probleme besser gelöst hätten als die Länder des 'real existierenden Sozialismus'. Im Rahmen der Abrüstungsvereinbarungen wurde nicht mehr mit den alten Propagandaparolen gearbeitet. Der Wunsch, zum 'gemeinsamen Haus Europa' zu gehören, mußte auch die früheren Vorwürfe gegenüber der Europäischen Gemeinschaft verdrängen.

Gewisse Schwierigkeiten gab es verständlicherweise bei der Darstellung der Verhältnisse in den sozialistischen Bruderstaaten. Bis in den Herbst 1989 hinein vermieden die sowjetischen Medien Kritik an diesen Bruderstaaten. Erst als ein Bruderstaat nach dem anderen den Sozialismus abschüttelte und schließlich die Berliner Mauer fiel, setzte sich (auch) in diesem Bereich die Wende durch.

Auf einmal war zu lesen, welche Fehler die einst dort herrschenden kommunistischen Parteien begangen hatten. Politische Persönlichkeiten, die eben noch gepriesen worden waren - so z. B. Honecker und Ceausescu wurden nun verhöhnt oder angegriffen. Den Sowjetbürgern wurde damit allerlei zugemutet. In kurzer Zeit mußten sie sich darauf umstellen, daß die eben noch als befreundet und auf ewig mit der Sowjetunion verbündeten Völker lieber heute als morgen die sowjetischen Streitkräfte aus dem Land haben wollten.

\section{Das alte Feindbild in den Streitkräften}

Nicht alle Feindbilder sind unter Gorbatschow verschwunden. Manche neue sind sichtbar geworden, die vorher von den Massenmedien verheimlicht worden waren. Gegen den Abbau der Feindbilder wehren sich besonders die sowjetischen Streitkräfte. In einer Handreichung des sowjetischen Verteidigungsministeriums, die 1988 in dritter Auflage herausgebracht wurde, wird das Feindbild als unverzichtbarer Bestandteil der politischen Erziehung herausgestellt. ${ }^{11}$ 
Genau so argumentiert ein Lehrbuch für die Presse der sowjetischen Streitkräfte vom Jahre 1989. Dort kann man u. a. lesen: „Nicht weniger wichtig sind die Materialien, die die militärischen Vorbereitungen und aggressiven Aktionen des Imperialismus aufzeigen, den Wesensgehalt der Militärdoktrinen aufdecken, die Mittel und Methoden der ideologischen Schulung des Personals der Armee der NATO im Geiste des Antikommunismus und Antisowjetismus ..."12.

Als der Vorschlag auftauchte, in den sowjetischen Streitkräften die politische Schulung zu beseitigen, haben sich die Streitkräfte mit allen Kräften dagegen gewehrt, und auf dem XXVIII. Parteitag 1990 haben sie dieses 'Erziehungsinstrument' erst einmal retten können. Damit wurde auch das Feindbild vom Kapitalismus und Imperialismus vorerst für einen Teil der Sowjetbürger bewahrt.

\section{Der schwierige Abschied vom Feindbild}

In der kühnen Zeitung 'Moskowskie nowostie' (Nr. 44/89) hat E. Ambarzumow einen Beitrag mit der Überschrift „Der schwierige Abschied vom Feindbild“ veröffentlicht. Er schreibt u. a.: „Das neue, von Michail Gorbatschow verkündete Denken, die von ihm immer beharrlicher hervorgehobene Einheit der jetzigen Welt zerstört - trotz aller Widersprüche - vor den Augen das Bild vom 'äußeren Feind'. Heißt das, daß überall das negative Vorzeichen in ein positives verändert werden muß? Es stellt sich heraus, daß das Feindbild noch nicht völlig aus dem gesellschaftlichen Bewußtsein verschwunden ist. Viele meinen bis heute, daß unsere derzeitige Unordnung wie früher damit zu erklären ist, daß der Feind uns schwächen und vernichten will. Für die einen sind es die Imperialisten, für andere die Zionisten und Freimaurer, für wieder andere alle zusammen.

Was uns angeht, so sollten wir uns auf die Worte des russischen Weisen Nikolai Berdjajew besinnen: 'Es ist eine geistige Lüge zu meinen, daß die Quelle alles Bösen sich außerhalb von mir befindet und ich selbst das Gefäß des Guten bin. ... Nein, die Quelle des Bösen befindet sich auch in mir selbst und ich muß auf mich Schuld und Verantwortung nehmen. ...' Diese Worte wurden 1924 ausgesprochen und seither wurde ihre Wahrheit vielfach bestätigt. Wenn wir Abschied nehmen vom Feindbild, dann gewinnen wir uns das menschliche Antlitz, die Menschenwürde zurück."

\section{Anmerkungen:}

1 Siehe hierzu: P. Roth, Die kommandierte öffentliche Meinung. Sowjetische Medienpolitik, Stuttgart 1982, $40 \mathrm{ff}$

2 Baedecker Rußland, Leipzig 1912, 284

3 J. Reed, 10 Tage, die die Welt erschütterten, Berlin (Ost) 1958, 327.

4 A. Abramov, U kremleskoj steny, Moskau 1983, 21.

5 Pamjatniki revoljucionnoj Rossii, Moskau 1986, 18.

6 Siehe hierzu: K. Schlögel, Moskau lesen, Berlin 1984, $45 \mathrm{f}$.

7 W. Leonhard, Die Revolution entläßt ihre Kinder, Köln/Berlin 1955, 31f.

8 S. Heym, Der König David Bericht, Gütersloh 1984, 116. 
9 Razrusennye i oskverennye chramy, Frankfurt/M. 1980, 151.

10 Memorial'nye asambli v gorodach- gerojach, Moskau 1985.

11 Ideologiceskaja rabota c casti: Soderzanie, organiszacija, metodika, Moskau 31988.

12 Voennaja pecat'. Kurs voennoj zurnalistiki. Ucebnoe posobie, Moskau 1989, 209.

\section{SUMMARY}

Images of foes and of friends, statues, icons (of a worldly and of a religious nature) are to be found in every society. In the Soviet Union the 'image monopoly' was in the hands of the political leadership. It was the latter which determined who was to be depicted as a friend, who as an enemy of the workers and of the nation; and who was to be honoured. Stalin and Chruschtschow both exchanged some of these images with their ideas. Gorbachev then began to replace these images in a fashion that can only be considered as sensational for the Soviet Union. Anti-religious propaganda was taken back, the foe image of imperialism/capitalism suppressed. However, the foe image imperialism lives on within the ranks of the Soviet armed forces. The above mentioned, desired replacement of images has partly been taken from the leadership's hands. Bold Soviet citizens are interfering, contradicting the leaders, attacking the, thus long, untouchable Lenin. The monument of the all-combining, all-uniting Communist Party seems to be beginning to crumble.

\section{RÉSUMÉ}

Des images d'ennemis et d'amis, des statues et des icônes (des caractère séculier ou religieuxé se trouvent dans chaque société. Dans l'Union soviétique le "monopole d'images" était exerce par les leaders politiques. Ceux-ci ont déterminé qui devait être considéré comme ami ou comme ennemi des ouvriers et du peuple, et finalement qui devait être honoré. Staline et Khrouchtchev ont échangé quelques-unes de ces images avec leurs idées. Puis Gorbatchev a commencé de remplacer ces images, un fait qu'on ne peut qualifier que de sensationnel pour l'Union soviétique. La propagande anti-religieuse a été supprimée, de même l'image d'ennemi que représentait l'impérialisme/capitalisme. Pourtant cette image existe toujours au sein des forces armées soviétiques. Ce remplacement désiré des images ne repose plus exclusivement entre les mains des leaders. Des citoyens audacieux dans l'U.R.S.S interviennent, ils contredisent les leaders et ils attaquent Lénine qui était intouchable pendant si longtemps. Il paraît que le monument du Parti Communiste embrassent tout et tous commence à s'écrouler

\section{RESUMEN}

Se pueden encontrar im genes de enemigos y de amigos, estatuas, iconos seculares o religiosos en todas las sociedades. En la Union Soviética el "monopolio de imagenes" se encontraba en las manos de los poderes politicos. Ellos determinaron a quien conviniera considerar como amigo o enemigo de los obreros y del pueblo y a quien conviniera honrar. Stalin y Kruschef confundieron algunas de esas imagenes con sus ideas. Gorbachov empez. Ó remplarar esas ideas de una manera que se puede elasificar solo como sensacional al considerar que se trata de la U.R. S.S.. Se suprimieron la propaganda antireligiosa y la imagen de enemigo del imperialismo/capitalismo. Sin embargo, esta imagen de enemigo sigue existiendo entre las fuerzas armadas soviéticas. La mencionada sustitucion deseada de las imagenes ya no est exclusivamente en las manos de los póderes y atacando a Lenin que ha sido considerado por tanto tiempo como intocable. Parece que el monumento del Partido Comunista, que une todo y a todos, empieza a caer en ruina. 